Editorial

\title{
Retraso en la cirugía del trauma vertebral y patologia compleja de columna frente a la Pandemia por Covid-19 en Chile y América Latina
}

\author{
Ratko Yurac B. ${ }^{1}$ Juan P. Cabrera C. ${ }^{2}$ Alfredo Guiroy ${ }^{3}$ Charles Carazzo ${ }^{4}$ Juan J. Zamorano ${ }^{1}$ \\ Bartolomé Marre ${ }^{1}$ Carlos Valderrama ${ }^{5}$ \\ ${ }^{1}$ Professor of Orthopedics and Traumatology, University del \\ Desarrollo (UDD), Clínica Alemana de Santiago, Chile \\ 2 Departamento de Neurocirugía, Hospital Clínico Regional de \\ Concepción, Chile \\ 3 Unidad de Columna Vertebral, Departamento de Ortopedia, \\ Hospital Español de Mendoza, Argentina \\ ${ }^{4}$ Profesor de Neurocirugía, Universidad de Campinas (UNICAMP), \\ Campinas, São Paulo, Brasil \\ ${ }^{5}$ Orthopedics and Traumatology Resident, University del Desarrollo \\ (UDD), Clínica Alemana de Santiago, Chile
}

Rev Chil Ortop Traumatol 2020;61:37-39.

La literatura ha sido creciente y diversa en el conocimiento sobre lo catastrófico de la pandemia por COVID-19. Sin lugar a duda ésta ha afectado fuertemente a casi a todos los países del mundo, contabilizando hasta ahora cerca de 14 millones de infectados y casi 600.000 muertes a nivel mundial. ${ }^{1}$ Como consecuencia la actividad médica se ha visto transversalmente alterada tanto en nuestro país, como en el resto de América Latina, donde elementos adicionales, como el invierno en curso y la precaria situación social que presenta la región, han facilitado la transmisión y diseminación del virus, convirtiéndonos hoy en el foco mundial de la pandemia. En muchos países latinoamericanos, los gobiernos establecieron cuarentenas totales para minimizar la exposición dentro de la población, en un intento de aplanar la curva epidemiológica de la enfermedad, con resultados variables, por las diferencias sociales, económicas y culturales de una región tan diversa. Por su parte, el personal de salud se ha dividido en grupos de trabajo para mejorar las condiciones laborales e intentar garantizar la cobertura de salud médica a las diferentes patologías y enfermedades, sin embargo, el COVID-19, ha saturado nuestros sistemas. Considerando además que los recursos son limitados, los sistemas de salud no han logrado la ambiciosa misión de detectar a todos los pacientes asintomáticos.

Por otro lado, antes de que el SARS-CoV-2 llegara a Latinoamérica, el momento ideal para la cirugía y tratamiento de los casos de trauma de la columna vertebral siempre estuvo muy influenciado por las condiciones locales, presentando gran heterogeneidad, con diferencias en el acceso y en la oportunidad de salud entre los sistemas público y privado. Esto motivó al grupo de estudio de trauma de AOSpine LatinAmerica (AOSLA Trauma Study Group) a realizar recientemente una encuestaen nuestra región para evaluar el retraso en el tratamiento de lesiones traumáticas de columna que requieren cirugía: "Retraso quirúrgico en fracturas toracolumbares inestables tipo B y C en América Latina. ¿Cuánto tiempo se tarda?". Al tratarse de lesiones con indicación quirúrgica absoluta, se espera evaluar cuál es el impacto del retraso de la cirugía en estos casos.

Los beneficios asociados a la cirugía precoz en trauma espinal se han demostrado en la literatura con estudios de alto nivel, tanto en casos de trauma de columna cervical ${ }^{2}$ como tóraco lumbar. ${ }^{3}$ En especial, los casos con déficit neurológico incompleto generalmente se deben tratar con mayor urgencia (como una emergencia quirúrgica) en comparación a aquellos con lesión medular completa, que deben manejarse como urgencia antes de 24 horas si el estado del paciente lo permite. ${ }^{4}$ La cirugía precoz (dentro de las primeras 24 horas) en lesiones traumáticas cervicales permite promover la recuperación neurológica (en especial en lesiones incompletas), lo que se asocia a significativos beneficios para el paciente ${ }^{5}$

Con respecto a la cirugía de columna durante la pandemia de COVID-19, diferentes sociedades relacionadas han establecido recomendaciones con el objetivo de realizar una cirugía de emergencia o confinada para pacientes con compresión severa de la raíz nerviosa, lesión de la médula espinal, agravamiento
Address for correspondence Ratko Yurac B, MD, Spine Surgeon

- Professor of Orthopedics and

Traumatology, University del

Desarrollo (UDD), Clínica

Alemana de Santiago, Chile

(e-mail: ryurac@gmail.com).
DOI https://doi.org/

10.1055/s-0040-1715521.

ISSN 0716-4548.
Copyright $\odot 2020$ by Thieme Revinter Publicações Ltda, Rio de Janeiro, Brazil
License terms

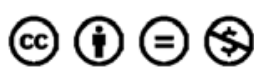


progresivo del déficit neurológico o fractura vertebral desplazada, inestable o con compresión severa del cordón medular o raíces nerviosas. ${ }^{6-9}$ Sin embargo, muchas de estas cirugías necesitan camas en unidades de cuidados intensivos(UCI) en el contexto de pacientes o cirugías de alto riesgo. Esta situación es particularmente frecuente en caso de politraumatismo, cuando habitualmente se requiere manejo integral prolongado en estas unidades, con monitorización estrecha para mantener presiones arteriales medias cercanas a $90 \mathrm{~mm} \mathrm{Hg}$ y oxigenación sobre $90-95 \%$ que permitan una adecuada oxigenación medular. Lamentablemente en este periodo de pandemia que hemos enfrentado y a pesar del importante aumento en el número total de camas UCI,éstas están completamente ocupadas o, de no estarlo, los pacientes que no estén cursando un cuadro producido por COVID-19 no son una prioridad para su uso en base a la proyección estimada de difusión del virus en especial en fase $3 .^{10}$ Esto basado en la información obtenida de realidades principalmente europeas y sin conocimiento del comportamiento que tendría el virus en Latinoamérica e influenciado por los patrones de comportamiento al comienzo de la pandemia en cada país específico.

Además, y nuevamente a consecuencia de la contingencia actual, la cirugía de columna no es la mayor preocupación para los administradores de los hospitales y no es una prioridad del gobierno durante este tiempo de pandemia. Sin embargo, los cirujanos de columna deben ofrecer un tratamiento oportuno a los pacientes para evitar secuelas, complicaciones o progresión de deformidades que serán mas complejas de resolver posteriormente. ${ }^{11,12}$ Por lo general, los pacientes con traumatismos en la columna vertebral son jóvenes, sanos y potencialmente con mucho que aportar a la sociedad, lo que refuerza aún más la importancia de un tratamiento precoz y adecuado. Desafortunadamente, cuando estos pacientes son diagnosticados con una lesión traumática de su columna, no hay conocimiento sobre su estatus de COVID-19 (el que ha variado en base a la evolución, fase de la pandemia y comportamiento ciudadano). En caso de pacientes COVID$19(+)$ operados por patología vertebral, se pueden afectar los resultados del tratamiento, ${ }^{13}$ pero también aumenta el riesgo de infección al que se expone al personal de salud que participa tanto en la cirugía, como en su cuidado perioperatorio.

Un posible efecto secundario de la pandemia de COVID-19 en el manejo del trauma espinal es el retraso en el tratamiento quirúrgico en pacientes con lesiones menos graves, los que pueden desarrollar deformidades tardías 0 deterioro neurológico, debido a los recursos limitados de atención médica.

En Latino américa, incluso previo a la pandemia, ya existían limitaciones de recursos, como implantes vertebrales adecuados, camas de UCI, disponibilidad de pabellón y equipos con experiencia en cirugía de columna vertebral. Probablemente estas limitaciones sólo han aumentado a raíz de la pandemia.

Por otro lado, una lista de espera más larga de pacientes con otras patologías de columna que precisan cirugías electivas (escoliosis, deformidades y patología degenerativa que requiere instrumentación) es un hecho y esperable, especialmente en los lugares de menores recursos, lo que muy probablemente se asociará a complicaciones y problemáticas de salud futuras, aún no consideradas.

Llegamos a la conclusión de que la pandemia de COVID19 , sin precedentes, afectará profundamente el cuidado de la columna vertebral incluyendo todo el espectro de enfermedades espinales, particularmente casos de trauma en ubicaciones con deficiencias estructurales, de recursos y de asistencia médica limitada. Por lo tanto, los cirujanos de columna en Chile y América Latina deben intentaranticipar estos problemas con los administradores de sus centros hospitalarios y previsionales de salud en un intento de minimizar el sufrimiento de los pacientes y mejorar así los resultados en este adverso escenario.

Son muy pocos los centros en Chile y la región que han sido capaces de desarrollar, en base a sus recursos e infraestructura habitualmente sobre el promedio latinoamericano, protocolos y sistemas libres de COVID-19 permitiendo continuar con el manejo de pacientes quirúrgicos con patología de emergencia, urgencia y electiva no postergable, junto con sistemas de cirugía ambulatoria, que la literatura mundial ya ha demostrado como factible y segura. ${ }^{14}$

Por un lado, en estos pocos centros, como el nuestro, esto ha permitido y más aún nos ha obligado a desarrollar protocolos quirúrgicos, consensuar y evaluar riesgos y beneficios de cirugía de columna en tiempos de COVID19 , con resultados alentadores, que no muestran las tasas de infección COVID-19 y mortalidad demostrada en estudios iniciales de la pandemia en otras regiones del mundo. 6,10 Quizás los resultados publicados en la literatura hasta ahora son secundarios a la falta de flujos diferenciados de pacientes (probables COVID-19 vs casos sintomáticos respiratorios), pabellones, personal y testeos previos.

Como ya se mencionó, la pandemia ha estimulado el desarrollo en nuestro país de la cirugía ambulatoria en columna, traumatológica y neuroquirúrgica. Un claro ejemplo en nuestro centro es la cirugía ambulatoria de la hernia discal lumbar, la que por múltiples razones no se realizaba previamente, demostrando como la pandemia ha cambiado paradigmas, destrabando las burocracias que la limitaban.

Estos temas serán tabulados y publicados a la brevedad. Estos protocolos desarrollados, en constantes mejoras y la literatura en el mundo nos permitirá desarrollar estrategias para reiniciar cirugías de más alta complejidad cuando la situación así lo permita.

\section{Bibliografia}

1 COVID-19 Dashboard by the Center for Systems Science and Engineering (CSSE) at Johns Hopkins University. Recuperado el 15 de Julio de 2020 en: https://www.arcgis.com/apps/opsdashboard/ index.html\#/bda7594740fd40299423467b48e9ecf6

2 Fehlings MG, Vaccaro A, Wilson JR, et al. Early versus delayed decompression for traumatic cervical spinal cord injury: results 
of the Surgical Timing in Acute Spinal Cord Injury Study (STASCIS). PLoS One 2012;7(02):e32037. Doi: 10.1371/journal.pone.0032037

3 Rath SA, Kahamba JF, Kretschmer T, Neff U, Richter HP, Antoniadis G. Neurological recovery and its influencing factors in thoracic and lumbar spine fractures after surgical decompression and stabilization. Neurosurg Rev 2005;28(01):44-52. Doi: 10.1007/ s10143-004-0356-3

4 Ter Wengel PV, Feller RE, Stadhouder A, et al. Timing of surgery in traumatic spinal cord injury: a national, multidisciplinary survey. Eur Spine J 2018;27(08):1831-1838. Doi: 10.1007/s00586-018-5551-y

5 Ter Wengel PV, De Haan Y, Feller RE, Oner FC, Vandertop WP. Complete Traumatic Spinal Cord Injury: Current Insights Regarding Timing of Surgery and Level of Injury. Global Spine J 2020;10(03):324-331. Doi: 10.1177/2192568219844990

6 Zou J, Yu H, Song D, Niu J, Yang H. Advice on Standardized Diagnosis and Treatment for Spinal Diseases during the Coronavirus Disease 2019 Pandemic. Asian Spine J 2020;14 (02):258-263. Doi: 10.31616/asj.2020.0122

7 Jain NS, Alluri RK, Schopler SS, Hah R, Wang JC. COVID-19 and Spine Surgery: A Review and Evolving Recommendations. Global SpineJournal. SAGE PublicationsLtd; 2020

8 Silva RT, Fogaça Cristante A, Martus Marcon R, Pessoa De Barros-Filho TE. Medical care for spinal diseases during the covid-19 pandemic. Vol. 75, Clinics. Universidade de Sao Paulo; 2020

9 Donnallylii CJ, Shenoy K, Vaccaro AR, Schroeder GD, Kepler CK. TriagingSpine Surgery in the COVID-19 Era [Internet]. 2020. Available from: www.clinicalspinesurgery.com

10 Shoukat A, Wells CR, Langley JM, Singer BH, Galvani AP, Moghadas SM. Projecting demand for critical care beds during COVID-19 outbreaks in Canada. 2020:1-8. Doi: 10.1503/cmaj.200457

11 Sornsa-Ard T, Niramitsantiphong A, Liawrungrueang W. Management of Traumatic Spinal Fracture in the Coronavirus Disease 2019 Situation. Asian Spine J 2020;14(03):385-387

12 Meyer M, Prost S, Farah K, et al. Spine Surgical Procedures during Coronavirus Disease 2019 Pandemic: Is It Still Possible to Take Care of Patients? Results of an Observational Study in the First Month of Confinement. Asian Spine J 2020;14(03):336-340www. asianspinejournal.org [Internet]

13 Lei S, Jiang F, Su W, et al. Clinical characteristics and outcomes of patients undergoing surgeries during the incubation period of COVID-19 infection. EClinicalMedicine 2020;000:100331. Doi: 10.1016/j.eclinm.2020.100331

14 Giorgi PD, Villa F, Gallazzi E, et al. The management of emergency spinal surgery during the COVID-19 pandemic in Italy. Bone Joint J 2020;102-B(06):671-676 